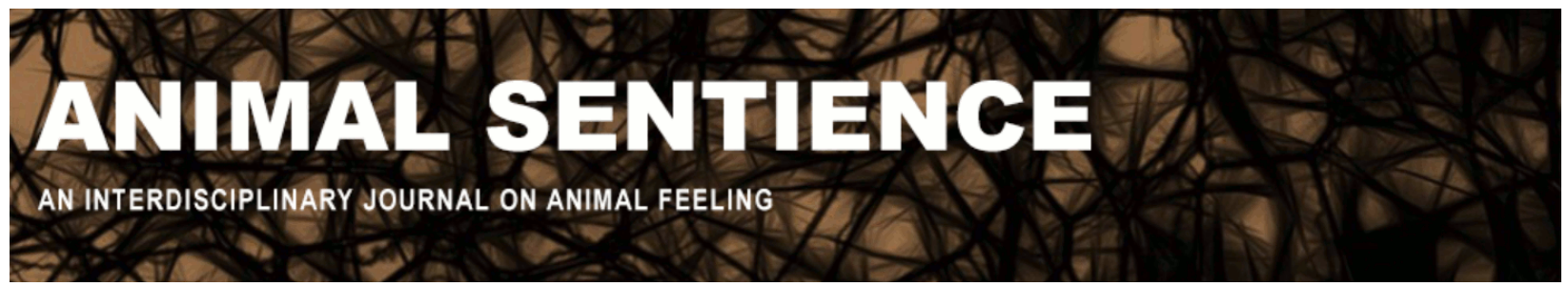

Key, Brian (2016) Phenomenal consciousness in insects? A possible way forward. Animal Sentience 9(17)

DOI: $10.51291 / 2377-7478.1159$

Date of submission: 2016-09-04 Date of acceptance: 2016-09-06 (c) (†) 


\title{
Phenomenal consciousness in insects? A possible way forward
}

Commentary on Klein \& Barron on Insect Experience

\author{
Brian Key \\ School of Biomedical Sciences \\ University of Queensland
}

\begin{abstract}
Klein \& Barron (2016) propose that subjective experience in humans arises in the midbrain and then argue that insects have the capacity for subjective experience because their nervous system can perform neural processing similar to that of the midbrain. This approach ultimately fails because it is built on the false premise that the midbrain is the source of the awareness of sensory stimuli. I instead propose that the capacity for subjective experience must be based on fundamental neural computations that generate the "what it feels like" experience. Two such computations associated with metarepresentations and high level representations entering working memory are discussed as possible measures of the capacity for subjective experience.
\end{abstract}

Keywords: subjective experience; phenomenal consciousness; metarepresentation; statistical summary

Brian Key is Head of the Brain Growth and
Regeneration Lab at the University of
Queensland. He is dedicated to understanding
the principles of stem cell biology,
differentiation, axon guidance, plasticity,
regeneration and development of the brain.
http://www.uq.edu.au/sbms/staff/brian-key

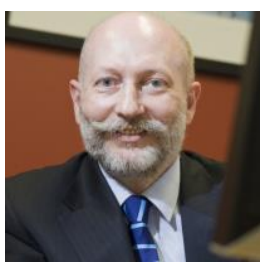

\section{Introduction}

No extant and scientifically plausible theory of phenomenal consciousness proposes that the midbrain is the site of subjective experience (e.g., Rolls, 2007; Lau and Rosenthal, 2011; Markov and Kennedy, 2013; Dehaene et al., 2014; Cleeremans et al., 2014; Koch et al., 2016; Wolf, 2016). The arguments for why the midbrain is not the generator of phenomenal consciousness have been clearly articulated (Key, 2015; Key, 2016a; Key, 2016b; Key, 2016c; Key, 2016d). To suggest, as do Klein \& Barron (2016), that the insect nervous system is capable of subjective experience because it appears to perform neural computations similar to those in the vertebrate midbrain is to ignore the weight of the contradictory evidence. Their conclusion about insect consciousness is no stronger than the proposition that any animal with a central nervous system has the capacity for subjective experience.

This is not to say that the approach adopted by Klein \& Barron is inappropriate. The idea that the search for subjective experience in animals should be based on neurobiological mechanisms is well founded. I have previously advocated this strategy and used it to reveal that fish lack the necessary neural architecture to support phenomenal consciousness (Key, 2016a; Key, 2016b). However, I have cautioned that this approach is more suitable for lineally related species with similar neurodevelopmental histories and neuroanatomical 
structures (Key, 2016). Attempting to apply neurobiological arguments, particularly those based on neuroanatomical structure, across widely disparate phyla such as Arthropoda and Chordata, and particularly between classes such as Insecta and Mammalia, leads only to weak inferences. The strength of Klein \& Barron's approach has been to search instead for functionally equivalent neural processing between insects and humans. As previously indicated (Key, 2016d), their approach fails because they inappropriately use nonconscious midbrain processing rather than conscious processing mechanisms in their assessment.

\section{Looking for Another Way Forward}

Given the disparate morphologies of human and insect nervous systems (Key, 2016e), an alternative approach for assessing subjective capacity is needed. A possible way forward is to identify neural computations (and generic circuit architectures capable of performing these computations) that are necessary for phenomenal consciousness. It has been proposed in several prominent theories of consciousness that the "what it is like" experience of a sensory stimulus requires an independent secondary observer neural network to introspect (i.e., to observe and interrogate) the signal processing within the neural network creating the primary representation of the sensory stimuli (Rolls, 2007; Cleeremans, 2014; Cruse and Shilling, 2015). This metarepresentation concept percolated out of philosophical discussions concerning the theory of mind (Leslie, 1987). However, in this context they were considered to be copies of the primary representation; it was only later that they were proposed to be representations of the underlying neural computations generating the representations of sensory stimuli (Singer, 1998; Cleeremans et al., 2007; Pasquali et al., 2010). Cleeremans et al. (2007) initially demonstrated with simple computer simulations that these observer neural networks were capable of associating the internal states of other independent networks with their future outcome. Because of this predictive power, the observer network could generate an expected outcome and could hence be said to be "cognizant" of what the network being monitored was processing. Thus, the observer network (most likely in the form of a recurrent neural network; Cruse, 2003) appears to possess the antecedent origins of awareness or subjective experience.

The field of "machine consciousness" is particularly interested in understanding the architecture necessary for creating phenomenal consciousness or what is referred to as "synthetic phenomenology" in robots (Chrisley, 2009). Computational models have been developed that use secondary neural networks to create metarepresentations of the primary neural networks that generate representations of sensory stimuli. Initial results are encouraging in that these computational models can generate conscious-like visual behaviours in robots that were not directly encoded for in the models (Arrabales et al., 2011).

Thus, there are now strong philosophical and empirical reasons to consider metarepresentations of neural computations generating sensory representations as a necessary property for a nervous system to be able to subjectively experience sensory stimuli. It is not required at this stage to define how metarepresentations generate subjective experience. For our goal of having a measure of the capacity for subjective experience, it is sufficient to merely propose that a phenomenally conscious nervous system must possess observer neural networks that are able to introspect and create 
metarepresentations of its sensory neural processing. While this would not be proof of subjective consciousness, it would provide insight into whether insects have (at least) one necessary neural property underlying subjective experience.

Working memory, or the ability to maintain neural representations in the absence of the initial stimulus that generated that activity in order to allow processing for the purpose of goal-directed behaviour, is believed to be essential for phenomenal consciousness (Baddeley, 1992; Osaka, 2016). Although all neural activity in working memory may not be conscious (Sato and Silvanto, 2014), there are some characteristics of the neural activity in working memory that seem necessary for subsequent progression into consciousness. Since the attentional capacity of working memory in humans is limited, it has been argued that low level representations of sensory stimuli (such as those in V1 visual cortex) are far too information-rich to enter into consciousness (Cohen et al., 2016). Higher order representations (such as those generated in the temporal lobe) of lower order representations are needed so as not to overload the attentional capacity of working memory and to provide a rich vivid mental image of the visual world. These higher order representations are proposed to be statistical summaries of the low level visual representations. I propose that if the insect nervous system is capable of visual consciousness, it must possess neural circuitry capable of generating higher order representations or statistical summaries of low level visual representations.

\section{Conclusion}

In summary, I have suggested that to begin to understand whether insects have the capacity for subjective experience it is essential to assess whether the insect nervous system has the neural architecture to perform computations that are recognised as fundamental for phenomenal consciousness. I have highlighted two such possible computations: (i) generating metarepresentations of sensory processing and (ii) creating high order representations of low level sensory representations in the form of statistical summaries. The challenge is to now identify the neural architecture(s) that can perform these computations so that we have simple tools for assessing the likelihood that insects are capable of subjective experience.

\section{References}

Arrabales, R., Ledezma, A. and Sanchis, A. (2011) Simulating visual qualia in the CERACRANIUM cognitive architecture. In From brains to systems (pp. 223-238). Springer: New York.

Baddeley, A. (1992) Working memory. Science 255:556-559.

Chrisley, R. (2009) Synthetic phenomenology. Inter. J. Mach. Conscious. 1:53-70.

Cleeremans, A. (2007) Consciousness and metapresentations: a computational sketch. Neural Net. 20:1032-1039.

Cleeremans, A. (2014) Connecting conscious and unconscious processing. Cogn. Sci. 38:12861315.

Cleeremans, A., Timmermans, B. and Pasquali, A. (2014) Connecting conscious and unconscious processing. Cogn. Sci. 38:1286-1315. 
Cohen, M.A., Dennett, D.C. and Kanwisher, N. (2016) What is the bandwidth of perceptual experience? Trends Cogn. Sci. 20:324-335.

Cruse, H. (2003) The evolution of cognition - a hypothesis. Cogn. Sci. 27:135-155.

Cruse, H. and Schilling, M. (2015) Mental states as emergent properties - from walking to consciousness. In T. Metzinger \& J. M. Windt (Eds.), Open MIND: 9(C). Frankfurt am Main: MIND Group. doi: 10.15502/9783958570436

Dehaene, S., Charles, L., King, J.-R. and Marti, S. (2014) Toward a computational theory of conscious processing. Curr. Opin. Neurobiol. 25:76-84.

Key, B. (2015) Fish do not feel pain and its implications for understanding phenomenal consciousness. Biol. Philos. 30:149-165.

Key, B. (2016a) Why fish do not feel pain. Animal Sentience 2016.003.

Key, B. (2016b) Going beyond just-so stories. Animal Sentience 2016.022.

Key, B. (2016c) Falsifying the null hypothesis that "fish do not feel pain". Animal Sentience 2016.039.

Key, B. (2016d) Burden of proof lies with the proposer of the celestial teapot hypothesis. Animal Sentience 2016.079.

Key, B. (2016e) Development and regeneration of the vertebrate brain. In G. Steinhoff (Ed.), Regenerative medicine - from protocol to patient ( $3^{\text {rd }}$ Edition, pp. 249-290). Springer: Switzerland.

Klein, C. and Barron, A.B. (2016) Insects have the capacity for subjective experience. Animal Sentience 2016.100.

Koch, C., Massimini, M., Boly, M. and Tononi, G. (2016) Neural correlates of consciousness: progress and problems. Nature Rev. Neurosci. 17:307-321.

Lau, H. and Rosenthal, D. (2011) Empirical support for higher-order theories of conscious awareness. Trends Cogn. Sci. 15:365-373.

Leslie, A.M. (1987) Pretense and representation: the origins of the "theory of mind". Psychological Rev. 94:412-426.

Markov, N.T. and Kennedy, H. (2013) The importance of being hierarchical. Curr. Opin. Neurobiol. 23:187-194.

Osaka, M. (2016) Working memory as a basis of consciousness. In Cognitive neuroscience robotics $B$ (pp. 39-57). Springer: Japan.

Pasquali, A., Timmermans, B. and Cleeremans, A. (2010) Know thyself: metacognitive networks and measures of consciousness. Cognit. 117:182-190.

Rolls, E.T. (2007) A computational neuroscience approach to consciousness. Neural Net. 20:962-982.

Soto, D. and Silvanto, J. (2014) Reappraising the relationship between working memory and conscious awareness. Trends Cogn. Sci. 18:520-525.

Wolf, S. (1998) Consciousness and the structure of neuronal representations. Phil. Trans. $R$. Soc. Lond. B 353:1829-1840. 